\title{
CANONICAL POLYADIC DECOMPOSITION OF COMPLEX-VALUED MULTI-WAY ARRAYS BASED ON SIMULTANEOUS SCHUR DECOMPOSITION
}

\author{
Sepideh Hajipour Sardouie ${ }^{(1,2,3)}$ Laurent Albera $^{(1,2)}$ Mohammad B. Shamsollahi $^{(3)}$ Isabelle Merlet $^{(1,2)}$ \\ ${ }^{(1)}$ Inserm, UMR 642, Rennes, F-35000, France $\quad{ }^{(2)}$ LTSI, University of Rennes 1, Rennes, F-35000, France \\ (3) BiSIPL, Sharif University of Technology, Tehran, Iran
}

\begin{abstract}
In this paper, we propose a new semi-algebraic algorithm to compute the Canonical Polyadic (CP) decomposition of complex-valued multi-way arrays. The proposed algorithm is based on the Simultaneous Schur Decomposition (SSD) of particular matrices derived from the array to process. This $\mathrm{CP}$ algorithm solves some convergence problems of classical iterative techniques and its identifiability assumptions are less restrictive than those of other semi-algebraic methods. We also propose a new Jacobi-like algorithm to calculate the SSD of several complex-valued matrices. Finally the usefulness of the proposed method is illustrated in the context of fluorescence spectroscopy and epileptic source localization.
\end{abstract}

Index Terms - Canonical Polyadic Decomposition, CANDECOMP/PARAFAC model, Simultaneous Schur Decomposition, Jacobi-like optimization, epileptic source localization.

\section{INTRODUCTION}

The Canonical Polyadic or CANDECOMP/PARAFAC (CP) decomposition consists in decomposing a Higher Order $(\mathrm{HO})$ array as a linear combination of a minimal number of rank- 1 terms. The $\mathrm{CP}$ decomposition can then be seen as a generalization of the Singular Value Decomposition (SVD) of two-way data to multi-way data. But the main difference is that, under weak assumptions [1], [2], no orthogonality constraint is needed to ensure uniqueness of the $\mathrm{CP}$ decomposition. This advantage makes the $\mathrm{CP}$ decomposition very useful in various applications [3], [4], [5], [6], [7], [8].

Several different methods were proposed to solve the CP problem. Generally, they can be divided into two categories: fully iterative and semi-algebraic methods. The fully iterative methods, such as the Alternative Least Squares (ALS) [9], Levenberg-Marquardt (LM) [10], [11] and nonlinear conjugate gradient approaches [12], try to estimate factors by starting from an initial point and iteratively decreasing the corresponding cost function. As the general iterative algorithms, these algorithms suffer from converging to local minima. To cope with this problem, different initial points must be used, which results to a time-consuming procedure. Unlike the iterative algorithms, the semi-algebraic algorithms do not require any initialization. These algorithms try to rewrite the $\mathrm{CP}$ problem into a more classical matrix problem such as joint eigenvalue decomposition [13], [14], simultaneous diagonalization by congruence [15] or Simultaneous Generalized Schur Decomposition (SGSD) [16], [17] of several matrices. These reformulated problems are usually solved by means of a Jacobi-like procedure.

In this paper, we propose a new semi-algebraic algorithm for $\mathrm{CP}$ decomposition of complex-valued $\mathrm{HO}$ arrays which is based on Simultaneous Schur Decomposition (SSD) of several matrices. Previously, a CP algorithm based on SGSD originally introduced in [18] was proposed in [16]. This CP algorithm aims at decomposing realvalued three-way arrays. In fact, as those proposed in [17], it consists in reformulating the $\mathrm{CP}$ problem into an unsymmetric simultaneous triangularization problem. To deal with the latter, two QR decompositions are assumed and the problem is solved by using a QZ iteration method followed by an algorithm to compute the triangular matrices (three different methods are introduced in [17]). Note that some constraints on the rank of the loading matrices must be assumed for identifiability of these algorithms [16], [17]. Compared with these $\mathrm{CP}$ algorithms, our formulation differs in such a way that results in a symmetric simultaneous triangularization problem. In addition, the proposed $\mathrm{CP}$ algorithm imposes no limitation on the order of the complex-valued arrays to decompose and the identification constraints are also less restrictive. Also a new SSD algorithm for decomposition of several complex-valued matrices is proposed. Finally the usefulness of the proposed method is illustrated in the context of fluorescence spectroscopy and epileptic source localization.

\section{METHODOLOGY}

In the following sections, bold uppercase letters (e.g. A), bold lowercase letters (e.g. $\boldsymbol{a}$ ) and calligraphic letters (e.g. $\mathcal{A}$ ) are used to denote matrices, vectors and $\mathrm{HO}$ arrays, respectively. The superscripts ${ }^{*},{ }^{\top},{ }^{\top}, \quad$ stand for the complex conjugate, the Moore-Penrose pseudoinverse, the transpose and the complex conjugated transpose, respectively.

\subsection{Complex Simultaneous Schur Decomposition}

In this section, we introduce a new Jacobi-like algorithm in order to calculate the SSD of several complex-valued matrices. It is a non trivial extension of a method proposed in the nineties for real-valued matrices only [19].

The SSD problem can be expressed as the following simultaneous triangularization problem. Given $R$ matrices $\boldsymbol{Y}^{(r)} \in \mathbb{C}^{d \times d}$ that have the following structure:

$$
\boldsymbol{Y}^{(r)}=\boldsymbol{\Theta}^{(o)} \boldsymbol{X}^{(r)} \Theta^{(o)^{\mathrm{H}}}
$$

where $\boldsymbol{\Theta}^{(o)} \in \mathbb{C}^{d \times d}$ is a unitary matrix and where the $R$ matrices $\boldsymbol{X}^{(r)} \in \mathbb{C}^{d \times d}$ are upper triangular matrices, our objective is the simultaneous triangularization of the $R$ matrices $\boldsymbol{Y}^{(r)}$ by finding the appropriate unitary matrix $\Theta^{(o)}$. In the presence of noise, the matrices $\boldsymbol{Y}^{(r)}$ do not exactly fit (1), so $\boldsymbol{\Theta}^{(o)}$ should be computed as an approximate solution to the problem of simultaneous upper triangularization. Indeed, we should find matrix $\Theta^{(o)}$ which minimizes the following cost function:

$$
\psi\left(\boldsymbol{\Theta}^{(o)}\right)=\sum_{r=1}^{R}\left\|\mathcal{L}\left(\boldsymbol{\Theta}^{(o)^{\mathrm{H}}} \boldsymbol{Y}^{(r)} \boldsymbol{\Theta}^{(o)}\right)\right\|_{F}^{2}
$$


where $\mathcal{L}\left(\boldsymbol{Y}^{(r)}\right)$ extracts the strictly lower triangular part of its matrixvalued argument by replacing the upper triangular part with zeros and where $\|\cdot\|_{F}$ denotes the Frobenius norm.

Minimizing the function $\psi$ in several variables is a hard optimization problem, except for small dimensions. The idea is to reformulate this multivariate optimization problem in a finite sequence of monovariate optimization problems using a Jacobi-like optimization scheme. Since any unitary matrix can be written as a product of elementary Givens rotation matrices, we can parameterize the orthogonal matrix $\boldsymbol{\Theta}^{(o)}$ as follows:

$$
\Theta^{(o)}=\prod_{s w=1}^{\# \text { of sweeps }} \prod_{q=1}^{d} \prod_{p=1}^{q-1} \Theta^{(o, p, q)}
$$

where each elementary Givens rotation matrix $\boldsymbol{\Theta}^{(o, p, q)}$ is obtained from an identity matrix in which we have $\Theta_{p, p}^{(p, q)}=c, \Theta_{p, q}^{(p, q)}=s$, $\Theta_{q, p}^{(p, q)}=-s^{*}$ and $\Theta_{q, q}^{(p, q)}=c$, where $(c, s) \in \mathbb{R} \times \mathbb{C}$ such that $c^{2}+|s|^{2}=1$. An appropriate parameterization of $c$ and $s$ in the complex domain used in the proposed algorithm is:

$$
s=\frac{t}{\sqrt{1+|t|^{2}}}, \quad c=\frac{1}{\sqrt{1+|t|^{2}}}, \quad t \in \mathbb{C}
$$

In each iteration, a new elementary Givens rotation matrix $\boldsymbol{\Theta}^{(o, p, q)}$ which defines $\boldsymbol{\Theta}^{(o)}$ (3) is computed in order to minimize the cost function (2). So, for each $p$ and $q$, the $R$ complex-valued matrices $\boldsymbol{Y}^{(r)}$ are transformed as:

$$
\boldsymbol{Y}^{(r) \prime}=\boldsymbol{\Theta}^{(o, p, q)^{\mathrm{H}}} \boldsymbol{Y}^{(r)} \boldsymbol{\Theta}^{(o, p, q)}
$$

Since this orthogonal similarity transformation changes only the elements of $\boldsymbol{Y}^{(r)}$ which appear in rows and columns $p$ and $q$, the change in the cost function can be expressed as:

$$
\begin{gathered}
\Delta \psi\left(\boldsymbol{\Theta}_{q, p}\right)=\left(1+|t|^{2}\right)^{-2}\left(v_{8}+2 \operatorname{Re}\left\{t\left(v_{3}^{*}-v_{9}^{*}\right)\right\}-2 \operatorname{Re}\left\{t^{2}\left(v_{4}^{*}\right)\right\}\right. \\
\left.\quad+|t|^{4} v_{6}+|t|^{2}\left(v_{1}+v_{10}-2 \operatorname{Re}\left\{v_{7}\right\}\right)+2 \operatorname{Re}\left\{t|t|^{2}\left(v_{5}^{*}-v_{2}^{*}\right)\right\}\right) \\
+\left(1+|t|^{2}\right)^{-1}\left(u_{1}-2 \operatorname{Re}\left\{t u_{2}\right\}+|t|^{2} u_{3}+w_{1}+2 \operatorname{Re}\left\{t w_{2}\right\}\right. \\
\left.\quad+|t|^{2} w_{3}\right)-\sum_{r=1}^{R}\left(\left|Y_{q, p}^{(r)}\right|^{2}+\sum_{k=p+1}^{q-1}\left(\left|Y_{k, p}^{(r)}\right|^{2}+\left|Y_{q, k}^{(r)}\right|^{2}\right)\right)
\end{gathered}
$$

where the coefficients $v_{1}-v_{10}, u_{1}-u_{3}$ and $w_{1}-w_{3}$ are defined in appendix. To calculate an appropriate complex value for $t$ in each iteration, we use the parameterization $t=\rho e^{\mathrm{i} \phi}$ and then we differentiate (6) with respect to $\rho$ and $\phi$ separately. Note that the roots of the following equation system are the stationary points of $\Delta \psi\left(\boldsymbol{\Theta}_{q, p}\right)$.

$$
\frac{\partial \Delta \psi(\rho, \phi)}{\partial \rho}=0 \quad \text { and } \quad \frac{\partial \Delta \psi(\rho, \phi)}{\partial \phi}=0
$$

It can be shown that by merging both equations of (7) and simplifying the resulting equation, the wanted $\phi$ value can be obtained by rooting a single 14-th degree polynomial in variable $x=e^{\mathrm{i} 2 \phi}$ whose coefficients $A_{\ell}$ are given in appendix. By rooting this polynomial numerically, fourteen complex values $\bar{x}_{k}$ are extracted. Now, we should choose the optimum solution $\bar{x}^{(o)}$ among all possible $\bar{x}_{k} \mathrm{~s}$ that minimizes the cost function (6). Since the equation (6) is a function of $t$, we should first calculate the corresponding value $t_{k}$ for each $\bar{x}_{k}$ in order to choose the best solution. To this end, we do the following computations.

By using the values $\bar{x}_{k}$, the corresponding values $\hat{\phi}_{k}$ can be computed as follows:

$$
\hat{\phi}_{k}=\frac{\operatorname{angle}\left\{\bar{x}_{k}\right\}}{2}, 1 \leq k \leq 14
$$

Afterwards for each $\hat{\phi}_{k}$, two corresponding values $\hat{\rho}_{k}^{(+)}$and $\hat{\rho}_{k}^{(-)}$ are calculated by rooting the second degree polynomial $\frac{\partial \Delta \psi\left(\rho, \hat{\phi}_{k}\right)}{\partial \hat{\phi}_{k}}$ in variable $\rho$ :

$\hat{\rho}_{k}^{(+/-)}=\frac{-\operatorname{Re}\left\{b_{1} e^{\mathrm{i} 2 \hat{\phi}_{k}}\right\} \pm \sqrt{\left(\operatorname{Re}\left\{b_{1} e^{\mathrm{i} 2 \hat{\phi}_{k}}\right\}\right)^{2}-4 \operatorname{Re}\left\{b_{0} e^{\mathrm{i} \hat{\phi}_{k}}\right\} \operatorname{Re}\left\{b_{2} e^{\mathrm{i} \hat{\phi}_{k}}\right\}}}{2 \operatorname{Re}\left\{b_{0} e^{\mathrm{i} \hat{\phi}_{k}}\right\}}$

where $b_{0}, b_{1}$ and $b_{2}$ are given in appendix.

So, for each $\bar{x}_{k}$, two couples $\left(\hat{\phi}_{k}, \hat{\rho}_{k}^{(+)}\right)$and $\left(\hat{\phi}_{k}, \hat{\rho}_{k}^{(-)}\right)$are obtained. Then, for each couple $\left(\hat{\phi}_{k}, \hat{\rho}_{k}^{(+/-)}\right), \hat{t}_{k}^{(+/-)}$is calculated as follows:

$$
\hat{t}_{k}^{(+/-)}=\hat{\rho}_{k}^{(+/-)} e^{\mathrm{i} \hat{\phi}_{k}}
$$

To select the optimum solution $t^{(o)}$, the cost function (6) is computed for the twenty-eight values $\hat{t}_{k}^{(+/-)}$and the argument of the minimum of (6) that makes it negative (to ensure that the cost function (2) is reduced in each step) is chosen as follows:

$$
t^{(o)}=\arg \min _{\hat{t}_{k}^{(+/)}} \Delta \psi\left(\boldsymbol{\Theta}_{q, p}\right)
$$

By using the selected $t^{(o)}, \boldsymbol{\Theta}^{(o, p, q)}$ is computed and the matrices $\boldsymbol{Y}^{(r)}$ are updated using (5).

\subsection{Canonical polyadic decomposition}

Let $\circ$ be the outer product operator [20], the CP decomposition of a $q$-th order complex-valued array $\mathcal{T}$ of size $\left(N_{1} \times N_{2} \times \ldots \times N_{q}\right)$ is defined by the following minimal linear combination:

$$
\mathcal{T}=\sum_{p=1}^{P} \boldsymbol{u}_{p}^{(1)} \circ \boldsymbol{u}_{p}^{(2)} \circ \cdots \circ \boldsymbol{u}_{p}^{(q)}
$$

where for each integer $i \in\{1, \ldots, q\}$ the $P$ complex-valued vectors $\boldsymbol{u}_{p}^{(i)}$ are the column vectors of a $\left(N_{i} \times P\right)$ matrix $\boldsymbol{U}^{(i)}$ called loading matrix. The aim of the CP problem is to determine the $q$ loading matrices $\boldsymbol{U}^{(i)}$ of $\mathcal{T}$ up to a diagonal scaling matrix and a permutation which are the classical indeterminacies of the $\mathrm{CP}$ decomposition.

Generally, people prefer handle unfolding matrices of multi-way arrays. All possible matricizations of a $q$-way array $\mathcal{T}$ can be defined by using two parameters, a permutation function $\pi$ and an integer $L \in\{1, \ldots, q\}$ :

$$
\begin{aligned}
& T(\pi, L)_{k, \ell}=\mathcal{T}_{n_{1}, n_{2}, \ldots, n_{q}} \\
& k=n_{\pi(1)}+\sum_{j=2}^{L}\left(n_{\pi(j)}-1\right) N^{(\pi(1), \pi(2), \ldots, \pi(j-1))} \\
& \ell=n_{\pi(L+1)}+\sum_{j=L+2}^{q}\left(n_{\pi(j)}-1\right) N^{(\pi(L+1), \pi(L+2), \ldots, \pi(j-1))}
\end{aligned}
$$

with $N^{\left(a_{1}, \ldots, a_{K}\right)}=N_{a_{1}} N_{a_{2}} \ldots N_{a_{K}}$. In the proposed algorithm, we use two different unfolding matrices, namely $\boldsymbol{T}^{(1)}=\boldsymbol{T}\left(\pi, L_{1}\right)$ and $\boldsymbol{T}^{(2)}=\boldsymbol{T}\left(\pi, L_{2}\right)$, where the permutation matrix $\pi$, the constants $L_{1}$ and $L_{2}\left(L_{1}<L_{2}\right)$ are defined in such a way that the smallest value of the three values $N^{\left(\pi(1), \pi(2), \ldots, \pi\left(L_{1}\right)\right)}$, $N^{\left(\pi\left(L_{1}+1\right), \pi\left(L_{1}+2\right), \ldots, \pi\left(L_{2}\right)\right)}$ and $N^{\left(\pi\left(L_{2}+1\right), \pi\left(L_{2}+2\right), \ldots, \pi(q)\right)}$, which is an upper bound of $P$, is the largest among all possible triples $\left(\pi, L_{1}, L_{2}\right)$. Indeed, our purpose is to propose an algorithm able to compute a $\mathrm{CP}$ decomposition with the maximal rank $P$. For the sake of simplicity, we assume in the following that $\pi$ is the identity permutation. 


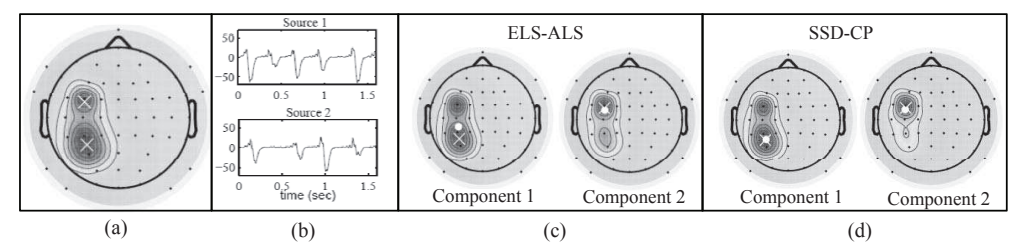

Fig. 1. An example of the localization process: (a) the location of two epileptic sources and the average potential distribution on the scalp, (b) the epileptic activity in the dipole sources of part (a), (c) the extracted components by using the ELS-ALS algorithm, (d) the extracted components by using the proposed algorithm. White crosses and white points represent the original and the estimated dipole locations respectively.

Then, it can be easily shown that $\boldsymbol{T}^{(1)}=\widetilde{\boldsymbol{U}}^{\left(L_{1}: 1\right)} \widetilde{\boldsymbol{U}}^{\left(q: L_{1}+1\right)^{\top}}$ with $\widetilde{\boldsymbol{U}}^{(k: l)}=\boldsymbol{U}^{(k)} \odot \boldsymbol{U}^{(k-1)} \odot \ldots \odot \boldsymbol{U}^{(l)}$ where $\odot$ denotes the Khatri-Rao product operator. Note that the $\widetilde{\boldsymbol{U}}^{\left(q: L_{1}+1\right)}$ matrix can also be written as follows:

$$
\begin{aligned}
\widetilde{\boldsymbol{U}}^{\left(q: L_{1}+1\right)^{\top}} & =\left(\widetilde{\boldsymbol{U}}^{\left(q: L_{2}+1\right)} \odot \widetilde{\boldsymbol{U}}^{\left(L_{2}: L_{1}+1\right)}\right)^{\top} \\
& =\left[\Phi^{(1)} \widetilde{\boldsymbol{U}}^{\left(L_{2}: L_{1}+1\right)^{\top}}, \ldots, \Phi^{\left(N^{*}\right)} \widetilde{\boldsymbol{U}}^{\left(L_{2}: L_{1}+1\right)^{\top}}\right]
\end{aligned}
$$

with $N^{*}=N^{\left(L_{2}+1, \ldots, q\right)}$ and where $\Phi^{(i)}$ is a $(P \times P)$ diagonal matrix for which diagonal is equal to the elements of the $i$-th row of $\widetilde{\boldsymbol{U}}^{\left(q: L_{2}+1\right)}$. By comparing the SVD $\boldsymbol{U} \boldsymbol{\Sigma} \boldsymbol{V}^{\mathrm{H}}$ of $\boldsymbol{T}^{(1)}$ truncated at order $P$ with the previous factorized expression of $\boldsymbol{T}^{(1)}$, we conclude that it exists a $(P \times P)$ non-singular matrix $\boldsymbol{W}$ such that:

$$
\boldsymbol{V}^{\mathrm{H}}=\boldsymbol{W} \widetilde{\boldsymbol{U}}^{\left(q: L_{1}+1\right)^{\mathrm{T}}}
$$

Now by inserting (14) into (15), we can rewrite $\boldsymbol{V}^{\mathrm{H}}$ as follows:

$$
\boldsymbol{V}^{\mathrm{H}}=\left[\boldsymbol{W} \Phi^{(1)} \widetilde{\boldsymbol{U}}^{\left(L_{2}: L_{1}+1\right)^{\top}}, \ldots, \boldsymbol{W} \Phi^{\left(N^{*}\right)} \widetilde{\boldsymbol{U}}^{\left(L_{2}: L_{1}+1\right)^{\top}}\right]
$$

Now let's define the $N^{*}$ matrices $\Gamma^{(i)}$ of size $\left(P \times N^{\left(L_{1}+1, \ldots, L_{2}\right)}\right)$ by $\boldsymbol{\Gamma}^{(i)}=\boldsymbol{W} \Phi^{(i)} \widetilde{\boldsymbol{U}}^{\left(L_{2}: L_{1}+1\right)^{\top}}$. By assuming that each matrix $\boldsymbol{\Gamma}^{(i)}$ is full row rank, all matrices $\boldsymbol{\Gamma}^{(i)}$ admit a Moore-Penrose matrix inverse $\boldsymbol{\Gamma}^{(i) \sharp}$. So, we define $N^{* 2}-N^{*}$ matrices $\boldsymbol{Y}^{(i, j)}$ by $\boldsymbol{Y}^{(i, j)}=$ $\boldsymbol{\Gamma}^{(i)} \boldsymbol{\Gamma}^{(j) \sharp}$. It can be shown that $\boldsymbol{Y}^{(i, j)}=\boldsymbol{W} \boldsymbol{D}^{(i, j)} \boldsymbol{W}^{-1}$ where, for each couple $(i, j), \boldsymbol{D}^{(i, j)}=\Phi^{(i)} \Phi^{(j) \sharp}$ is a diagonal matrix. Thus, let us write $\boldsymbol{W}=\boldsymbol{Q R}$, namely the QR decomposition of matrix $\boldsymbol{W}$ where $\boldsymbol{Q} \boldsymbol{Q}^{\mathrm{H}}=\boldsymbol{I}$ and $\boldsymbol{R}$ is an upper triangular matrix. Then we can rewrite $\boldsymbol{Y}^{(i, j)}$ as follows:

$$
\boldsymbol{Y}^{(i, j)}=\boldsymbol{Q} \boldsymbol{R} \boldsymbol{D}^{(i, j)} \boldsymbol{R}^{-1} \boldsymbol{Q}^{-1}=\boldsymbol{Q} \boldsymbol{X}^{(i, j)} \boldsymbol{Q}^{\mathrm{H}}
$$

where the matrices $\boldsymbol{X}^{(i, j)}=\boldsymbol{R} \boldsymbol{D}^{(i, j)} \boldsymbol{R}^{-1}$ are upper triangular matrices. Since the elements on the diagonal of $\boldsymbol{X}^{(i, j)}$ are equal to the diagonal components of $\boldsymbol{D}^{(i, j)}$, by applying the proposed SSD algorithm to matrices $\boldsymbol{Y}^{(i, j)}$, the upper triangularized matrices $\boldsymbol{X}^{(i, j)}$ and consequently the diagonal matrices $\boldsymbol{D}^{(i, j)}$ are extracted. Now let's see how the components of $\widetilde{\boldsymbol{U}}^{\left(q: L_{2}+1\right)}$ can be identified from those of the matrices $\boldsymbol{D}^{(i, j)}$. Since $\boldsymbol{D}^{(i, j)}=\Phi^{(i)} \Phi^{(j) \sharp}$, for each column of $\widetilde{\boldsymbol{U}}^{\left(q: L_{2}+1\right)}$, we have $R=N^{* 2}-N^{*}$ equations. If we define $\boldsymbol{U}^{*}=\widetilde{\boldsymbol{U}}^{\left(q: L_{2}+1\right)}$, for column $p$, we have:

$$
\forall i, j, i \neq j, \quad \frac{U_{i, p}^{*}}{U_{j, p}^{*}}=D_{p, p}^{(i, j)} \Rightarrow U_{i, p}^{*}-D_{p, p}^{(i, j)} U_{j, p}^{*}=0
$$

These equations can be written in a matrix form:

$$
\boldsymbol{M}^{(p)} \boldsymbol{u}_{p}^{*}=\mathbf{0}_{R \times 1}
$$

where $\boldsymbol{u}_{p}^{*}$ is the $p$-th column of matrix $\boldsymbol{U}^{*}$ and the matrix $\boldsymbol{M}^{(p)}$ of size $\left(\left(N^{* 2}-N^{*}\right) \times N^{*}\right)$ is defined as follows. For each ordered pair $\left\{(i, j), i \neq j, 1 \leq i, j \leq N^{*}\right\}$, we consider one row in matrix $\boldsymbol{M}^{(p)}$ such that the element in the $i$-th column is equal to 1 , the element in the $j$-th column is equal to $-D_{p, p}^{(i, j)}$ and the other elements are 0 . Note that the order of the rows are unimportant in the solution. It is also noteworthy that the system (19) has an infinite number of solutions due to the scaling indeterminacies of the CP decomposition. So we set the elements of the first row of $\boldsymbol{U}^{*}=\widetilde{\boldsymbol{U}}^{\left(q: L_{2}+1\right)}$ equal to one and then we extract the elements of the other rows from (19). Now, since we have $\boldsymbol{T}^{(2)}=\widetilde{\boldsymbol{U}}^{\left(L_{2}: 1\right)} \widetilde{\boldsymbol{U}}^{\left(q: L_{2}+1\right)^{\top}}$, by assuming the full column rank of $\widetilde{\boldsymbol{U}}^{\left(q: L_{2}+1\right)}, \widetilde{\boldsymbol{U}}^{\left(L_{2}: 1\right)}$ can be calculated as follows:

$$
\widetilde{\boldsymbol{U}}^{\left(L_{2}: 1\right)}=\boldsymbol{U}^{\left(L_{2}\right)} \odot \ldots \odot \boldsymbol{U}^{(1)}=\boldsymbol{T}^{(2)}\left(\pi, L_{2}\right)\left(\widetilde{\boldsymbol{U}}^{\left(q: L_{2}+1\right)^{\top}}\right)_{(20)}^{\sharp}
$$

Next, the $\left(N_{L_{2}} \times \ldots \times N_{1}\right)$ rank-1 array $\widetilde{\mathcal{U}}_{p}^{\left(L_{2}: 1\right)}$ associated to the $p$-th column $\boldsymbol{u}^{(p)}$ of $\widetilde{\boldsymbol{U}}^{\left(L_{2}: 1\right)}$ is generated as follows:

$$
\widetilde{\mathcal{U}}_{p, i_{L_{2}}, \ldots, i_{2}, i_{1}}^{\left(L_{2}: 1\right)}=u_{i_{L_{2}}+\sum_{i=i_{L_{2}-1}}^{i_{1}}\left((i-1) N^{\left(L_{2}, \ldots, i+1\right)}\right)}^{(p)}(
$$

Then by computing a simple rank-1 HOSVD [21] of $\widetilde{\mathcal{U}}_{p}^{\left(L_{2}: 1\right)}$, the $p$-th columns of matrices $\boldsymbol{U}^{(1)}, \ldots, \boldsymbol{U}^{\left(L_{2}\right)}$ are extracted. The same procedure can be applied to the matrix $\widetilde{\boldsymbol{U}}^{\left(q: L_{2}+1\right)}$ to extract the other loading matrices $\boldsymbol{U}^{(j)}$ for $j \in\left\{L_{2}+1, \ldots, q\right\}$.

\section{SIMULATIONS}

\subsection{Multiway analysis of amino acids fluorescence data}

In this section, we examine the effect of overfactoring, say an overestimation of the array rank, on the performance of different $\mathrm{CP}$ methods. To this end, we use the Amino acids fluorescence data downloaded from [22] and described in [22], [23]. This data set comprises five simple laboratory-made samples. Each sample is composed of different amounts of tyrosine, tryptophan and phenylalanine dissolved in phosphate buffered water. The samples were measured by fluorescence on a PE LS50B spectrofluorometer with excitation slit-width of $2.5 \mathrm{~nm}$, an emission slit-width of $10 \mathrm{~nm}$ and a scan-speed of $1500 \mathrm{~nm} / \mathrm{s}$ [22]. Since each individual amino acid gives a rank-1 contribution to the data, ideally we can describe these data with three CP components [22]. We applied three classical CP methods, namely ALS [9], ELS-ALS [10], LM [10] and the SSD-CP method proposed in this paper, to canonically decompose the amino acids fluorescence data. Then we compared the emission-mode factors estimated by the four methods by varying the rank $P$ of the $\mathrm{CP}$ decomposition from 3 to 5 as shown in Fig.2. Note that, regarding the three fully iterative methods, they are run several times with different random initial guess in order to use the best initialization. 


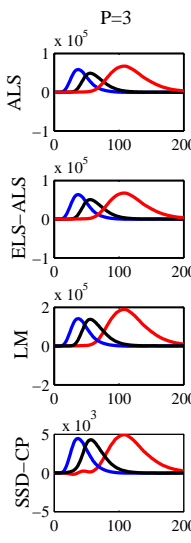

(a)
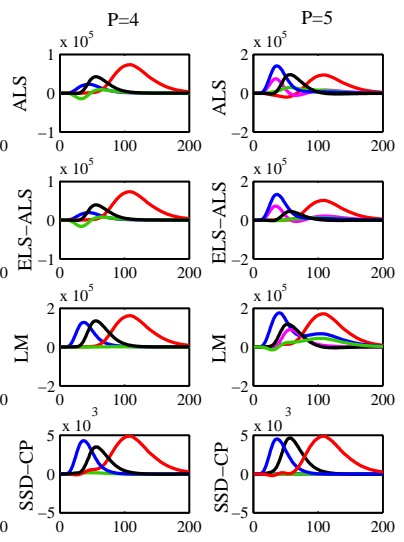

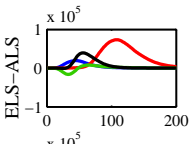

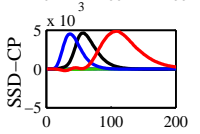

(c)
Fig. 2. The emission mode factors with the estimated number of components equal to (a) $P=3$, (b) $P=4$ and (c) $P=5$

As displayed in Fig.2(a), for $P=3$, all $\mathrm{CP}$ algorithms generate almost the same emission factors corresponding to three amino acids. As shown in Fig.2(b-c), in the case of $P=4$ and $P=5$, the factors obtained from the three iterative algorithms change, however the SSD-CP proposed technique generates three factors that match with the true factors while the forth and the fifth estimated factors are very small. These results show that SSD-CP is robust with respect to overfactoring.

\subsection{Localization of Epileptic Sources}

In this section, the efficiency of the proposed algorithm in extracting loading matrices of complex-valued arrays is studied in the context of the localization of epileptic Electroencephalographic sources. A 3-shell spherical head model, two superficial, radically oriented dipole sources (with random positions) and the Jansen model [24] are considered to construct the 64 channels of the epileptic data [7]. Then, the STFT of these data is used to construct the $\mathrm{HO}$ array $\mathcal{T} \in \mathbb{C}^{N_{1} \times N_{2} \times N_{3}}$ where three dimensions represent time, frequency and channel respectively. By using the $\mathrm{CP}$ algorithms, the location of the dipoles is estimated by extracting the corresponding factors. The efficiencies of the proposed algorithm and the ELS-ALS algorithm to estimate the location of the sources are compared in 100 monte-carlo trials. In each trial, we calculate the Root Mean square Error (RMSE) [7] between the estimated and the original locations of the sources. Fig.1 (a) shows the location of two epileptic sources and the average potential distribution on the scalp while Fig.1 (b) displays the epileptic activity of these dipole sources. Fig.1 (c) and (d) show the original and the estimated locations of the sources by using the ELS-ALS algorithm [20] and the proposed algorithm, respectively. In this figure, white crosses and white points represent the original and the estimated dipole locations, respectively. The average localization errors of the proposed algorithm and the ELS-ALS algorithm are 2.53 and $2.78 \mathrm{~cm}$, respectively.

\section{CONCLUSION}

In this paper, a new semi-algebraic SSD-based algorithm to compute the $\mathrm{CP}$ decomposition of multi-way complex-valued arrays was proposed. We also proposed a new Jacobi-like algorithm to calculate SSD of several complex-valued matrices. We compared the efficiency of the proposed algorithm with the ELS-ALS algorithm in the context of source localization. We showed that the localization error of the proposed algorithm is lower than that of the ELS-ALS algorithm, concluding that our algorithm can be used in the source localization context. We also showed that the proposed method is robust against the overestimation of the number of factors.

\section{APPENDIX}

To calculate the values of coefficients $A_{\ell}$, first we define the values $v_{1}$ to $v_{10}, u_{1}$ to $u_{3}$ and $w_{1}$ to $w_{3}$ as functions of elements of matrices $\boldsymbol{Y}^{(r)}, 1 \leq r \leq R$ as follows:

$$
\begin{aligned}
v_{1}= & \sum_{r=1}^{R}\left|Y_{p, p}^{(r)}\right|^{2}, v_{2}=\sum_{r=1}^{R} Y_{p, p}^{(r)} Y_{p, q}^{(r)}, v_{3}=\sum_{r=1}^{R} Y_{p, p}^{(r)} Y_{q, p}^{(r)^{*}} \\
v_{4}= & \sum_{r=1}^{R} Y_{p, p}^{(r)} Y_{q, q}^{(r)^{*}}, v_{5}=\sum_{r=1}^{R} Y_{p, q}^{(r)} Y_{q, q}^{(r)^{*}}, v_{6}=\sum_{r=1}^{R}\left|Y_{p, q}^{(r)}\right|^{2} \\
v_{7}= & \sum_{r=1}^{R} Y_{p, q}^{(r)} Y_{q, p}^{(r)^{*}}, v_{8}=\sum_{r=1}^{R}\left|Y_{q, p}^{(r)}\right|^{2}, v_{9}=\sum_{r=1}^{R} Y_{q, p}^{(r)} Y_{q, q}^{(r)^{*}} \\
v_{10}= & \sum_{r=1}^{R}\left|Y_{q, q}^{(r)}\right|^{2}, u_{1}=\sum_{r=1}^{R} \sum_{k=p+1}^{q-1}\left|Y_{k p}^{(r)}\right|^{2}, w_{1}=\sum_{r=1}^{R} \sum_{k=p+1}^{q-1}\left|Y_{q k}^{(r)}\right|^{2} \\
u_{2}= & \sum_{r=1}^{R} \sum_{k=p+1}^{q-1} Y_{k p}^{(r)} Y_{k q}^{(r)}, w_{2}=\sum_{r=1}^{R} \sum_{k=p+1}^{q-1} Y_{q k}^{(r)} Y_{p k}^{(r) *} \\
u_{3}= & \sum_{r=1}^{R} \sum_{k=p+1}^{q-1}\left|Y_{k q}^{(r)}\right|^{2}, w_{3}=\sum_{r=1}^{R} \sum_{k=p+1}^{q-1}\left|Y_{p k}^{(r)}\right|^{2}
\end{aligned}
$$

Then we define $b_{0}-b_{4}, c_{0}-c_{4}, t_{0}-t_{7}$ and $s_{0}-s_{5}$ as follows:

$b_{0}=j\left(v_{5}^{*}-v_{2}-u_{2}+w_{2}\right), b_{1}=-j 2 v_{7}^{*}, b_{2}=j\left(v_{3}^{*}-v_{9}-v_{2}+w_{2}\right)$

$b_{3}=-2 v_{1}+4 v_{6}+4 \operatorname{Re}\left\{v_{4}\right\}-2 v_{10}+2 u_{3}-2 u_{1}+2 w_{3}-2 w_{1}$

$b_{4}=2 v_{1}-4 \operatorname{Re}\left\{v_{4}\right\}-4 v_{8}+2 v_{10}+2 u_{3}-2 u_{1}+2 w_{3}-2 w_{1}$

$c_{0}=c_{4}^{*}=b_{1}^{2}, c_{1}=c_{3}^{*}=-4 b_{2}^{*} b_{0}^{*}, c_{2}=2\left|b_{1}\right|^{2}-8 \operatorname{Re}\left\{b_{2} b_{0}^{*}\right\}$

$t_{0}=t_{7}^{*}=2 j b_{2}^{*} b_{1}^{* 2} b_{0}^{* 2}-2 j b_{0} b_{1}^{* 4}-2 j b_{1}^{* 2} b_{0}^{* 3}+b_{3} b_{1}^{* 3} b_{0}^{*}$

$t_{1}=t_{6}^{*}=-4 j b_{2}^{* 2} b_{0}^{* 3}+4 j b_{2}^{*} b_{0}^{* 4}-4 j b_{2} b_{1}^{* 2} b_{0}^{* 2}+b_{0} b_{3} b_{1}^{* 3}$

$+2 j b_{1} b_{1}^{* 3} b_{0}^{*}+b_{4} b_{1}^{*} b_{0}^{* 3}+12 j b_{0} b_{2}^{*} b_{1}^{* 2} b_{0}^{*}-3 b_{3} b_{2}^{*} b_{1}^{*} b_{0}^{* 2}$

$t_{2}=t_{5}^{*}=10 j b_{0}^{2} b_{2}^{*} b_{1}^{* 2}-16 j b_{0} b_{2}^{* 2} b_{0}^{* 2}+6 j b_{0}^{2} b_{1}^{* 2} b_{0}^{*}-6 j b_{1} b_{0} b_{1}^{* 3}$

$+8 j b_{2} b_{0}^{* 4}+4 j b_{2} b_{2}^{*} b_{0}^{* 3}+4 j b_{0} b_{2}^{*} b_{0}^{* 3}-6 j b_{1} b_{1}^{*} b_{0}^{* 3}+3 b_{1} b_{3} b_{1}^{* 2} b_{0}^{*}$

$-2 j b_{1} b_{2}^{*} b_{1}^{*} b_{0}^{* 2}-3 b_{2} b_{3} b_{1}^{*} b_{0}^{* 2}+3 b_{0} b_{4} b_{1}^{*} b_{0}^{* 2}-6 b_{0} b_{3} b_{2}^{*} b_{1}^{*} b_{0}^{*}$

$t_{3}=t_{4}^{*}=8 j b_{2}^{2} b_{0}^{* 3}+4 j b_{0}^{3} b_{1}^{* 2}-12 j b_{0}^{2} b_{2}^{*} b_{0}^{* 2}-20 j b_{0}^{2} b_{2}^{* 2} b_{0}^{*}$

$+6 j b_{1}^{2} b_{1}^{* 2} b_{0}^{*}+20 j b_{2} b_{0} b_{0}^{* 3}+b_{1} b_{4} b_{0}^{* 3}+4 j b_{2} b_{0}^{2} b_{1}^{* 2}-3 b_{0}^{2} b_{3} b_{2}^{*} b_{1}^{*}$

$+3 b_{1} b_{0} b_{3} b_{1}^{* 2}+4 j b_{2} b_{0} b_{2}^{*} b_{0}^{* 2}-14 j b_{2} b_{1} b_{1}^{*} b_{0}^{* 2}-6 b_{2} b_{0} b_{3} b_{1}^{*} b_{0}^{*}$

$-6 j b_{1} b_{0} b_{1}^{*} b_{0}^{* 2}-3 b_{1} b_{3} b_{2}^{*} b_{0}^{* 2}+3 b_{0}^{2} b_{4} b_{1}^{*} b_{0}^{*}+12 j b_{1} b_{0} b_{2}^{*} b_{1}^{*} b_{0}^{*}$

$s_{0}=s_{5}^{*}=-2 j b_{0} b_{1}^{* 3}-2 j b_{1}^{*} b_{0}^{* 3}+b_{3} b_{1}^{* 2} b_{0}^{*}+2 j b_{2}^{*} b_{1}^{*} b_{0}^{* 2}$

$s_{1}=s_{4}^{*}=b_{4} b_{0}^{* 3}+b_{0} b_{3} b_{1}^{* 2}-4 j b_{2} b_{1}^{*} b_{0}^{* 2}+2 j b_{1} b_{1}^{* 2} b_{0}^{*}$ $-b_{3} b_{2}^{*} b_{0}^{* 2}+8 j b_{0} b_{2}^{*} b_{1}^{*} b_{0}^{*}$

$s_{2}=s_{3}^{*}=-4 j b_{1} b_{0}^{* 3}-4 j b_{1} b_{0} b_{1}^{* 2}-b_{2} b_{3} b_{0}^{* 2}+3 b_{0} b_{4} b_{0}^{* 2}-2 b_{0} b_{3} b_{2}^{*} b_{0}^{*}$ $+6 j b_{0}^{2} b_{2}^{*} b_{1}^{*}+6 j b_{0}^{2} b_{1}^{*} b_{0}^{*}-4 j b_{2} b_{0} b_{1}^{*} b_{0}^{*}+2 b_{1} b_{3} b_{1}^{*} b_{0}^{*}$

Finally, we can calculate the fifteen coefficients $A_{\ell}$ according to the predefined parameters:

$$
A_{\ell}=\sum_{k=\max (0, \ell-7)}^{\min (7, \ell)} t_{k} t_{\ell-k}-\sum_{i=\max (0, \ell-9)}^{\min (5, \ell)} \sum_{j=\max }^{\min (5, \ell-\ell-9, \ell-i-4)} s_{i} s_{j} c_{\ell-i-j}
$$




\section{REFERENCES}

[1] J. B. Kruskal, "Three-way arrays: Rank and uniqueness of trilinear decompositions," Linear Algebra and Applications, vol. 18, no. 2, pp. 95-138, 1977.

[2] T. Jiang and N. Sidiropoulos, “Kruskal's permutation lemma and the identification of CANDECOMP/PARAFAC and bilinear models with constant modulus constraints," IEEE Transactions on Signal Processing, vol. 52, no. 9, pp. 2625-2636, September 2004.

[3] J. Carroll and J. Chang, "Analysis of individual differences in multidimensional scaling via an n-way generalization of eckart-young decomposition," Psychometrika, vol. 35, no. 3, pp. 283-319, September 1970.

[4] R. Bro, "PARAFAC, tutorial and applications," Chemom. Intel. Lab. Syst., vol. 38, no. 2, pp. 149-171, October 1997.

[5] A. Villringer and B. Chance, "Noninvasive optical spectroscopy and imaging of human brain function," Trends Neuroscience, vol. 20, pp. 435-442, 1997.

[6] M. De Vos, A. Vergult, L. De Lathauwer, W. De Clercq, S. V. Huffel, P. Dupont, A. Palmini, and W. V. Paesschen, "Canonical decomposition of ictal EEG reliably detects the seizure onset zone," NeuroImage, vol. 37, no. 3, pp. 844-854, Septembre 2007.

[7] H. Becker, P. Comon, L. Albera, M. Haardt, and I. Merlet, "Multiway Space-Time-Wave-Vector analysis for source localization and extraction," in European Signal Processing Conference, Aalborg, Denmark, August 23-27 2010, pp. 1349_ 1353.

[8] H. Becker, P. Comon, L. Albera, M. Haardt, and I. Merlet, "Multi-way Space-Time-Wave-Vector analysis for EEG source separation," Elsevier Signal Processing, vol. 92, no. 4, pp. 1021-1031, April 2011.

[9] R.A. Harshman and M. E. Lundy, "PARAFAC: Parallel factor analysis," Computational Statistics and Data Analysis, vol. 18, no. 1, pp. 39-72, August 1994.

[10] P. Comon, X. Luciani, and A. L. F. De Almeida, "Tensor decompositions, alternating least squares and other tales," Journal of Chemometrics, vol. 23, no. 7-8, pp. 393-405, April 2009.

[11] G. Tomasi and R. Bro, "A comparison of algorithms for fitting the parafac model," Computational Statistics \& Data Analysis, vol. 50, no. 7, pp. 1700-1734, April 2006.

[12] E. Acar, D.M. Dunlavy, and T.G. Kolda, "A scalable optimization approach for fitting canonical tensor decompositions," Chemometrics, vol. 25, no. 2, pp. 67-86, February 2011.

[13] F. Roemer and M. Haardt, "A closed-form solution for multilinear parafac decompositions," in SAM 08, Fifth IEEE Sensor Array and Multichannel Signal Processing Workshop, july 2123 2008, pp. 487-491.

[14] X. Luciani and L. Albera, "Semi-algebraic canonical decomposition of multi-way arrays and joint eigenvalue decomposition," in IEEE International Conference on Acoustics Speech and Signal Processing, Prague, Czech Republic, May 22-27 2011, pp. 4104-4107.
[15] L. De Lathauwer, "A link between the canonical decomposition in multilinear algebra and simultaneous matrix diagonalization," SIAM Journal in matrix Analysis and Applications, vol. 28, no. 3, pp. 642-666, December 2006.

[16] L. De Lathauwer, B. De Moor, and J. Vandewalle, "Computation of the canonical decomposition by means of a simultaneous generalized Schur decomposition," SIAM Journal on Matrix Analysis and Applications, vol. 26, no. 2, pp. 295-327, November 2004

[17] M. Sørensen and L. De Lathauwer, "New simultaneous generalized Schur decomposition methods for the computation of the canonical polyadic decomposition," in Signals, Systems and Computers (ASILOMAR), 2010 Conference, Monterey, USA, November 7-10 2010, pp. 13-17.

[18] A.J. Van Der Veen and A. Paulraj, "An analytical constant modulus algorithm," IEEE Trans. Signal Process., vol. 44, no. 5, pp. 1136-1155, May 1996.

[19] M. Haardt and J.A. Nossek, "Simultaneous Schur decomposition of several nonsymmetric matrices to achieve automatic pairing in multidimensional harmonic retrieval problems," IEEE Transactions on Signal Processing, vol. 46, no. 1, pp. 161-169, January 1998.

[20] A. Karfoul, L. Albera, and L. De Lathauwer, "Iterative methods for the canonical decomposition of multi-way arrays: Application to blind underdetermined mixture identification," $E l$ sevier Signal Processing, vol. 91, no. 8, pp. 1789-1802, August 2011.

[21] L. De Lathauwer, B. De Moor, and J. Vandewalle, "A multilinear singular value decomposition," SIAM Journal on Matrix Analysis and Applications, vol. 21, no. 4, pp. 1253-1278, June 2000.

[22] R. Bro, "Parafac: Tutorial and applications," Chemometrics and Intelligent Laboratory Systems, , no. 38, pp. 149-171, 1997.

[23] R. Bro, Multi-way Analysis in the Food Industry: Models, Algorithms, and Applications, Ph.D. thesis, University of Amsterdam, Amstrdam, Netherlands, 1998.

[24] B. H. Jansen and V. G. Rit, "Electroencephalogram and visual evoked potential generation in a mathematical model of coupled cortical columns," Biological Cybernetics, vol. 73, no. 4, pp. 357-366, September 1995. 